\title{
Estudo comparativo da análise de Bolton pelos métodos manual e computadorizado
}

\author{
Comparative study of Bolton analysis \\ between manual and computerized methods \\ Estudio comparativo de los métodos de análisis de Bolton \\ manual y computarizado \\ Renato BIGLIAZZI ${ }^{\mathbf{1}}$ \\ Ricardo Luiz de Carvalho FERNANDEZ ${ }^{2}$ \\ Helga Adachi Medeiros BARBOSA ${ }^{2}$ \\ Derly Tescaro Narciso de OLIVEIRA ${ }^{3}$ \\ André Pinheiro de Magalhães BERTOZ ${ }^{\mathbf{1}}$ \\ Kurt FALTIN JUNIOR ${ }^{2}$
}

\author{
${ }^{1}$ Faculdade de Odontologia, UNESP Univ. Estadual Paulista, 16015-050 Araçatuba - SP, Brasil \\ ${ }^{2}$ Faculdade de Odontologia, UNIP Universidade Paulista, 04026-002 São Paulo - SP, Brasi \\ ${ }^{3}$ Faculdade de Odontologia da Universidade Brasil, 15600-000 Fernandópolis - SP, Brasil
}

\begin{abstract}
Resumo
O objetivo deste estudo foi avaliar a aplicabilidade do método computadorizado na obtenção da análise de Bolton e compará-lo com os métodos convencionais (Paquímetro Digital e Compasso de Ponta Seca). Foram utilizados 30 pares de modelos de gesso das arcadas dentárias de pacientes oriundos do curso de especialização da Universidade Paulista - FOUNIP, escolhidos aleatoriamente e em perfeito estado. Medidas manuais foram obtidas com paquímetro digital e com compasso de ponta seca e, por meio do programa computadorizado Radiocef®. Cada paciente teve os seus dentes medidos por três vezes nos diferentes métodos em intervalos de uma semana. Os dados foram analizados pela Análise de Variância (ANOVA) à um nível de significância de 5\% para se verificar a repetibilidade dos métodos e pela análise de Regressão para estudar a correlação entre os mesmos. Os métodos mais precisos foram os manuais sendo o que utiliza o Paquímetro Digital o mais preciso. Todas as Análises de Regressão foram estatisticamente significantes, ou seja, houve correlação entre os métodos. Analisando os resultados podemos notar que as melhores correlações foram obtidas entre os métodos Compasso de Ponta Seca e Paquímetro Digital. Pode-se concluir que o método mais preciso foi o Paquímetro Digital, sendo este 3 vezes mais preciso que os demais métodos.

Descritores: Dentição Permanente; Modelos Dentários; Ortodontia.
\end{abstract}

\begin{abstract}
The aim of this study was to evaluate the applicability of the computerized method for obtaining the Bolton analysis and compare it with conventional methods (Digital Caliper and Needlepoint Dividers). A total of 30 pairs of plaster models of the dental arches of patients from the specialization course of Universidade Paulista - FOUNIP, chosen at random and in perfect condition. Manual measurements were obtained with a digital caliper and needle point and through the Radiocef ${ }^{\circledR}$ computer program. Each patient had their teeth three times measured by the different methods in one-week intervals. Data were analyzed by analysis of variance (ANOVA) at a 5\% significance level to verify the repeatability of the methods and the regression analysis to study the correlation between them. The most accurate methods are manual and which uses the Digital Caliper more accurate. All regression analyzes were statistically significant, ie correlation between the methods. Analyzing the results we can see that the best correlations were obtained between the methods Needlepoint Dividers and Digital Caliper. It can be concluded that the most accurate method was the Digital Caliper, which is 3 times more accurate than other methods.
\end{abstract}

Descriptors: Dentition, Permanent; Dental Models; Orthodontics.

\section{Resumen}

El objetivo de este estudio fue evaluar la aplicabilidad del método informatizado para obtener el análisis de Bolton y compararlo con los métodos convencionales (calibrador digital y Ponta brújula Seca). Un total de 30 pares de modelos de yeso de las arcadas dentales de los pacientes del curso de especialización de la Universidad Paulista FOUNIP, elegido al azar y en perfectas condiciones. Las medidas manuales se obtuvieron con un espesor y una aguja de punta digital y a través del programa informático Radiocef®. Cada paciente tenía sus dientes tres veces medidos por los diferentes métodos en intervalos de una semana. Los datos fueron analizados por análisis de varianza (ANOVA) con un nivel de significación del 5\% para verificar la repetibilidad de los métodos y el análisis de regresión para estudiar la correlación entre ellos. Los métodos más precisos son manual y que utiliza el calibrador digital más preciso. Todos los análisis de regresión fueron estadísticamente significativos, es decir, la correlación entre los métodos. El análisis de los resultados, podemos ver que se obtuvieron los mejores correlaciones entre los métodos Ponta Seca brújula y un calibrador digital. Se puede concluir que el método más exacto fue el Caliper Digital, que es 3 veces más preciso que otros métodos.

Descriptores: Dentición Permanente; Modelos Dentales; Ortodoncia.

\section{INTRODUÇÃO}

O tratamento ortodôntico é fundamentado no diagnóstico que envolve a análise facial, o estudo cefalométrico e dos modelos, permitindo a avaliação dos padrões funcionais e musculares, esqueléticos e dentários. A análise dos modelos é frequentemente utilizada para a mensuração de tamanho, forma e posições dentárias. A identificação da presença de discrepância do tamanho dentário entre os arcos da maxila e da mandíbula é um importante componente do diagnóstico ortodôntico, influenciando o plano de tratamento quanto à redução ou 
aumento interproximal ou na indicação de exodontias ${ }^{1,2}$.

Bolton $^{3,4}$ desenvolveu um método de calcular a razão entre o diâmetro mesiodistal dos dentes da maxila e da mandíbula e afirmou que a correta e harmoniosa oclusão é possível somente quando existe adequada proporção entre o tamanho dos dentes.

Tradicionalmente, os índices de Bolton são medidos manualmente com o auxílio de um compasso de ponta seca ou um paquímetro em modelos de gesso ${ }^{5}$. A Ortodontia atualmente incorpora avanços também no campo do diagnóstico, através da tecnologia de digitalização de imagens (radiografias, modelos, fotografias, etc.). Esta tecnologia vem facilitar o dia a dia na clínica ortodôntica, melhorando tanto a rapidez como a precisão dos diagnósticos, incluindo a análise de modelos ${ }^{6}$. Portanto este estudo tem o objetivo de comparar a precisão de um método computadorizado na obtenção da Análise de Bolton e compará-la com os métodos convencionais (compasso de ponta seca e paquímetro digital).

\section{MATERIAL E MÉTODO}

Este estudo recebeu aprovação do Comitê de Ética em Pesquisa em Seres Humanos da Universidade Paulista FOUNIP antes do seu início. (067/09/CEP/ICS/UNIP). Foram utilizados 30 pares de modelos de estudo ortodônticos, superiores e inferiores, do arquivo de pacientes do curso de especialização da Universidade Paulista FOUNIP. Todos os pacientes eram leucodermas, com dentição permanente completa, de ambos os gêneros, com idade variando entre 12 e 20 anos, brasileiros e residentes na cidade de São Paulo. O critério de inclusão usado na seleção dos modelos foi a boa qualidade dos modelos, todos os dentes permanentes totalmente erupcionados, presença do segmento primeiro molar direito - primeiro molar do lado oposto, nenhuma extração dentária e ausência de desgastes interproximais e/ou oclusais. Foram excluídos os modelos com dentes quebrados, restaurações grosseiras, coroas, onlays, restaurações de classe II de amálgama ou restaurações compostas que afetassem o diâmetro mésiodistal dos dentes.

A metodologia utilizada neste estudo seguiu os preceitos preconizados por Bolton ${ }^{3,4}$, tanto para os métodos manuais (compasso de ponta seca e paquímetro digital) quanto para o método computadorizado. A medição manual do diâmetro mésio-distal dos dentes entre os pontos de contato ou no seu maior diâmetro individualmente, de primeiro molar permanente de um lado ao dente homólogo do lado oposto nos modelos, foi realizada com paquímetro digital Mitutoyo ${ }^{\circledR}$ (modelo PC-5G, Mitutoyo America Corporation, Illinois, USA) e com compasso de ponta seca Miltex ${ }^{\circledR}$ (modelo 68-695, Integra LifeSciences Corporation, Plainsboro, NJ, USA) e régua milimetrada Desetec $^{\circledR}$.(Trident@ $\odot$, Itapuí, São Paulo, Brasil). Para verificar o erro intraexaminador, cada paciente teve os seus dentes medidos por três vezes nos diferentes métodos. Executou-se a Análise de Bolton para cada indivíduo, calculando-se a soma do maior diâmetro mésio-distal dos dentes para obtenção tanto da discrepância total dos arcos (DT), quanto para a discrepância dos segmentos anteriores dos arcos (DA).

Para a medição computadorizada utilizou-se o programa de diagnóstico ortodôntico Radiocef ${ }^{\circledR}$ (Radiomemory, Belo Horizonte, Minas Gerais, Brasil), que segue uma sequência predeterminada para a medição dos diâmetros mesiodistais dos dentes envolvidos na análise. A digitalização dos modelos para a marcação dos pontos foi feita no Scanner Fotográfico HP Scanjet G4050 a 100\%, e 75 DPI de resolução, por critérios preconizados pelo software. Foram marcados os pontos na face oclusal dos modelos onde a marcação dos mesmos foi visualizada no monitor (Figura 1), e repetida três vezes pelo mesmo operador (como nos métodos manuais), utilizando-se o software. Executou-se a Análise de Bolton para cada indivíduo automaticamente, pois o software realiza todas as operações com extrema rapidez.

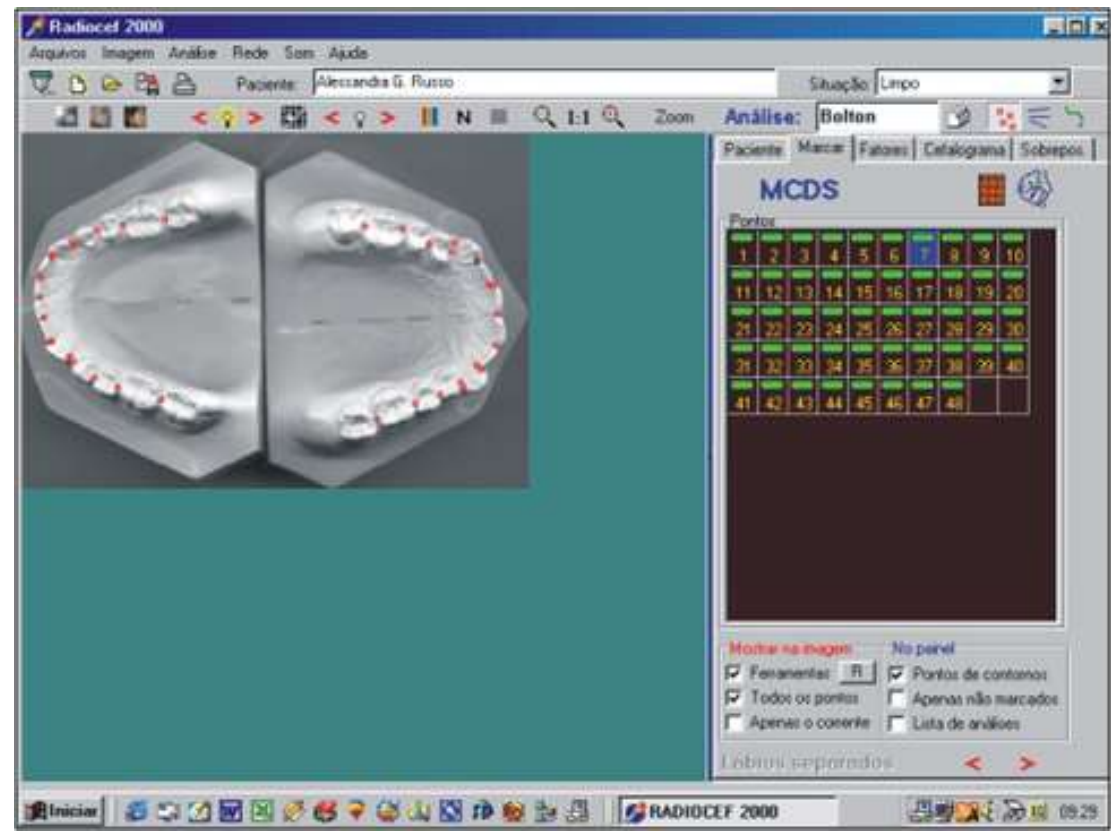

Figura 1: Método computadorizado.

\section{○ Análise Estatística}

Todas as três mensurações foram realizadas com intervalos de uma semana pelo mesmo examinador (RLCF). A média e o desvio padrão (DP) foram calculados para os valores obtidos para os DT e DA para toda a amostra, nos três métodos de medição. Para verificar o grau de repetitividade e precisão dos diferentes métodos nas mensurações dos dentes em todos os modelos, nos diferentes momentos de observação, foi utilizada a Análise de Variância (ANOVA) à um nível de significância de 5\%, obtendo-se assim o desvio padrão relativo ao método. Para se determinar o grau de correlação entre os métodos utilizouse a Análise de Regressão.

\section{RESULTADOS}

As Figuras 2 e 3 demonstram as variações dos resultados da DT e DA. A precisão dos métodos foi verificada através da "variabilidade média" entre as medidas de cada paciente utilizando a Análise de Variância $(\mathrm{P}<0,000)$. $\mathrm{O}$ valor da precisão é dado pelo quadrado médio da Repetitividade (ou Resíduo) e utiliza como expressão da precisão dois desvios-padrão para que possamos trabalhar com a porcentagem. A expressão do valor da precisão do método é a raiz quadrada deste valor (repetitividade ou Resíduo) multiplicada por 2 e quanto mais distante de zero menos preciso foi o método. Como podemos observar na Tabela 1, o método Paquímetro foi o mais preciso, apresentando uma precisão quase três vezes maior que os outros dois métodos. Além disto, observamos que os métodos compasso e computadorizado apresentaram precisão semelhante, com o compasso sendo um pouco mais preciso. As Tabelas 2, 3 e 4 apresentam a Análise de Regressão e verificamos que todas as correlações que fizemos foram significativas, ou seja, a correlação entre os métodos foi diferente de zero. Analisando os resultados nas tabelas, podemos notar que a melhor correlação foi obtida entre o método Compasso de Ponta Seca e Paquímetro Digital, indicando uma boa correlação entre eles. 


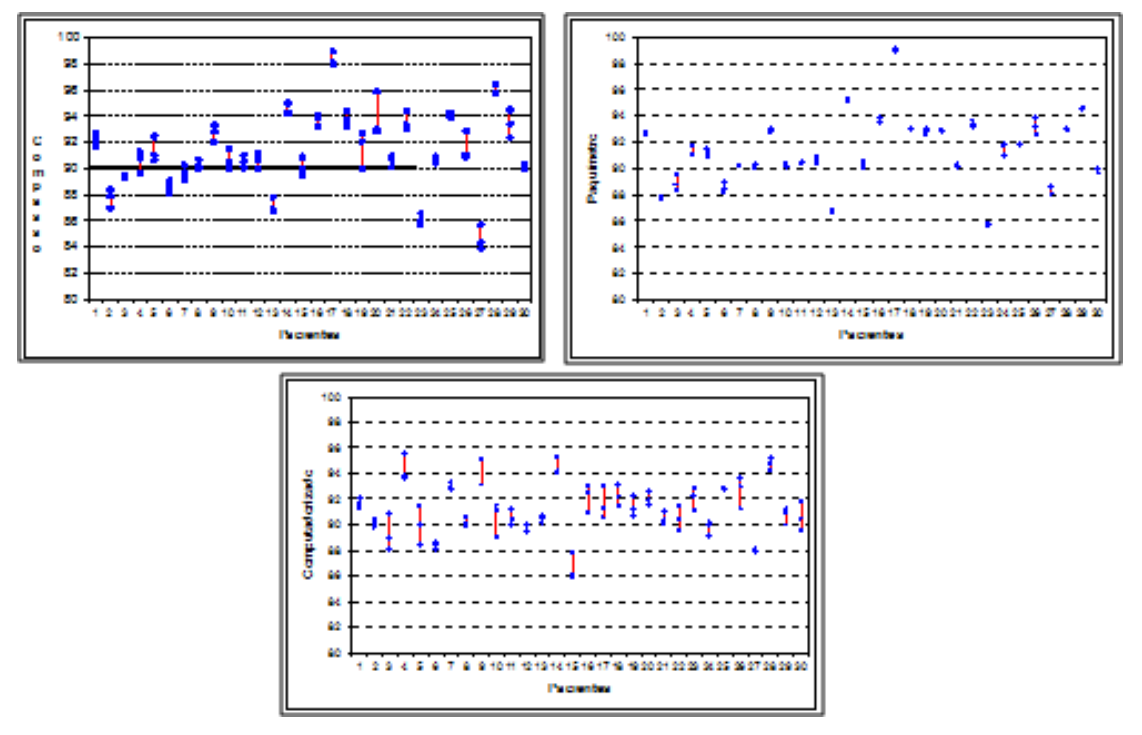

Figura 2: Variação dos resultados dos métodos para a variável DT.

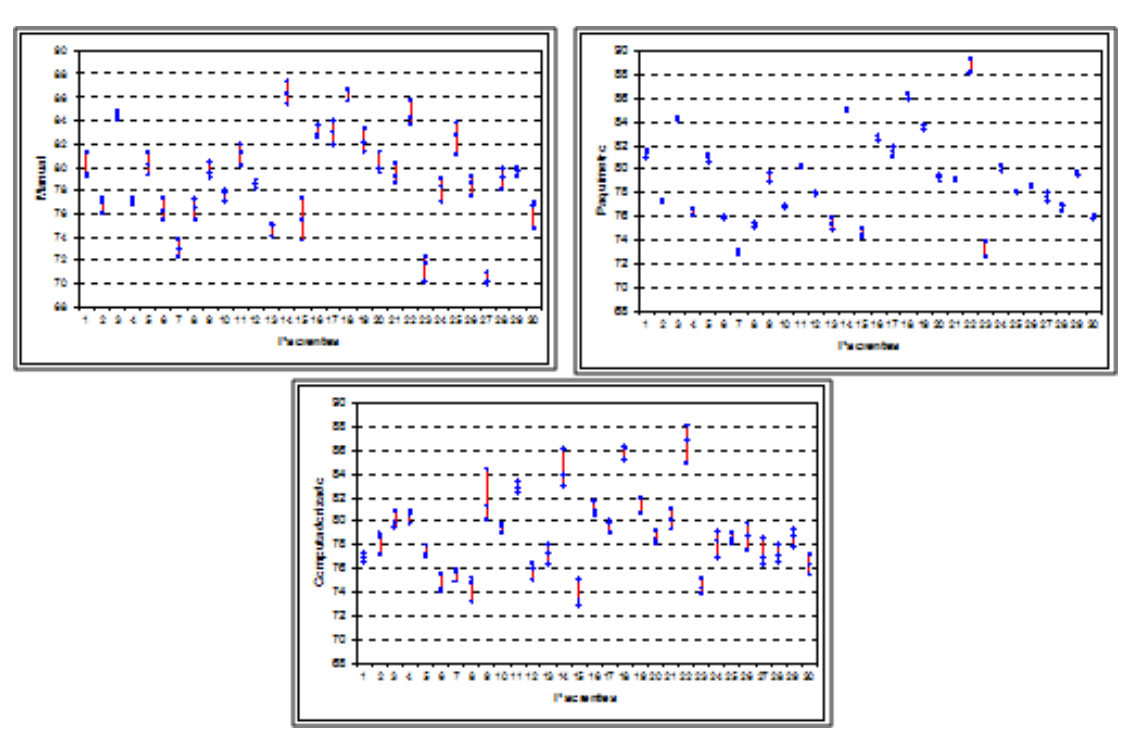

Figura 3: Variação dos resultados dos métodos para a variável DA.

Tabela 1. Valores da precisão do método

\begin{tabular}{c|cc}
\hline Método & DT & DA \\
\hline Compasso & 1,46 & 1,80 \\
Paquímetro & 0,50 & 0,62 \\
Computadorizado & 1,68 & 1,88 \\
\hline
\end{tabular}

Tabela 2. Comparação entre os métodos: compasso e paquímetro

\begin{tabular}{c|cc}
\hline & DT & DA \\
\hline Erro Médio & 0,81 & 1,34 \\
SEP & 1,26 & 2,15 \\
Erro máximo & 3,78 & 7,23 \\
\hline Correlação & $\mathbf{0 , 9 1 6}$ & $\mathbf{0 , 8 7 1}$ \\
\hline $\mathbf{R}^{\mathbf{2}}$ & 0,839 & 0,759 \\
Regressão & Compasso $=0,86+0,99 \times$ & Compasso $=6,78+0,91 \times$ \\
& Paquímetro & Paquímetro \\
\hline
\end{tabular}

Tabela 3. Comparação entre os métodos: compasso x computadorizado

\begin{tabular}{c|cc}
\hline & DT & DA \\
\hline Erro Médio & 1,77 & 1,96 \\
SEP & 2,56 & 2,46 \\
Erro máximo & 6,68 & 6,90 \\
\hline Correlação & $\mathbf{0 , 5 2 4}$ & $\mathbf{0 , 7 6 5}$ \\
\hline $\mathbf{R}^{2}$ & 0,275 & 0,585 \\
Regressão & Compasso $=19,13+$ & Compasso $=5,17+0,94 \times$ \\
& $0,99 \times$ Computadorizado & Computadorizado \\
\hline \multicolumn{2}{|c}{}
\end{tabular}

Tabela 4. Comparação entre os métodos: paquímetro e computadorizado

\begin{tabular}{c|cc}
\hline & DT & DA \\
\hline Erro Médio & 1,79 & 1,34 \\
SEP & 2,65 & 1,59 \\
Erro máximo & 7,33 & 2,66 \\
\hline Correlação & $\mathbf{0 , 5 2 4}$ & $\mathbf{0 , 8 3 8}$ \\
\hline $\mathbf{R}^{\mathbf{2}}$ & 0,275 & 0,703 \\
Regressão & Paquímetro $=36,02+0,61 \times$ & $\begin{array}{c}\text { Paquímetro }=1,87+0,98 \times \\
\text { Computadorizado }\end{array}$ \\
\hline
\end{tabular}

\section{DISCUSSÃO}

Há concordância na literatura quanto à necessidade de se tentar predizer a presença ou não de discrepâncias no tamanho dentário ${ }^{7-10}$. A obtenção de uma boa oclusão funcional com trespasses vertical e horizontal normais requer a adequada proporção do tamanho dos dentes da maxila e da mandíbula. Se existir uma discrepância interarcos do tamanho dentário, uma oclusão ideal como meta do tratamento ortodôntico, pode não ser alcançada, da mesma forma a quantidade de sobremordida e sobressaliência estão diretamente relacionadas com o tamanho dental ${ }^{7,10}$.

Desde a publicação da análise da discrepância do tamanho dentário ${ }^{3}$, vários autores ${ }^{4-6}$ sugerem conclusivamente a utilização dessa análise antes de se iniciar o tratamento ortodôntico. As medidas dos dentes feitas no modelo são mais acuradas e fáceis de serem obtidas do que diretamente no paciente; assim como as medidas feitas com o paquímetro são mais precisas do que com o compasso de ponta seca ${ }^{10,11}$,como verificado em nosso estudo. Shellhart et al. ${ }^{11}$ em 1995 avaliaram a confiabilidade da Análise de Bolton e concluiram que erros de medidas clinicamente significantes podem ocorrer quando essa análise é usada em pacientes com mais de $3 \mathrm{~mm}$ de apinhamento. Por outro lado, Halazonetis ${ }^{12}$ em 2005 observou que erros de medidas nos modelos não têm grande influência nos resultados e sim, a espessura vestíbulo-lingual dos incisivos.

A literatura é concordante que a discrepância no tamanho dentário é frequente, apesar das desarmonias na posição dos dentes não serem necessariamente uma consequência dessas discrepâncias. Com a revolução tecnológica dos computadores acelerada a partir da década de 80, houve a popularização de programas ortodônticos computadorizados para tentar dinamizar e acelerar a coleta e interpretação dos dados utilizados rotineiramente no diagnóstico ortodôntico e ortopédico facial ${ }^{13-16}$.

O presente estudo foi elaborado para determinar se os dois métodos manuais (paquímetro digital e compasso de ponta seca) e o computadorizado são acurados e eficientes quando se realiza a análise da discrepância do tamanho dentário. Para tal utilizamos uma amostra de pacientes brasileiros leucodermas, ambos os gêneros, com dentição permanente e que apesar de necessitarem de tratamento ortodôntico ainda não haviam se submetido ao mesmo.

Nossos resultados são claros em demonstrar a maior precisão do paquímetro digital, que é cerca de três vezes mais preciso que os outros dois métodos, sendo que entre os métodos computadorizado e compasso de ponta seca, o segundo apresentou um pouco mais de precisão. A análise de regressão verificou que os métodos, paquímetro digital e compasso de ponta seca, se assemelham mais e indicou uma boa correlação entre eles, enquanto o método computadorizado foi menos preciso.

Existe concordância na literatura quanto à confiabilidade da análise da discrepância do tamanho dentário, quanto à precisão do paquímetro digital e sua correlação com a medição pelo compasso de ponta seca ${ }^{6,11}$. A utilização de fotocópia de modelos ortodônticos para a medição dentária não se mostra tão precisa certamente pela perda da tridimensionalidade ${ }^{11,12,17,18}$. Somente Tomassetti et $\mathrm{al}^{6}$, utilizando um sistema de medição conectado diretamente ao computador, obtiveram resultado semelhante ao método de medição do paquímetro digital, tendo como vantagens a precisão e a rapidez do software, que não incorpora erros de cálculo. Tomassetti et $\mathrm{al}^{6}$ encontraram também que $72.7 \%$ das medidas diminuiam cerca de $1.0 \mathrm{~mm}$ 
entre as medições ( 0 to $2.8 \mathrm{~mm}$ ) quando a Análise de Bolton foi calculada em três tempos diferentes com o paquímetro digital diretamente nos modelos de estudo.

A Análise de Bolton não resulta em excelente reprodutibilidade e uma das principais razões que justificam a divergência entre os métodos manual e digital, segundo Brandão et $\mathrm{al}^{19}$, é que os pontos de referência podem ser de difícil localização e a opinião do examinador sobre a localização exata de um ponto pode variar aleatoriamente ${ }^{20}$. Brandão et al $^{19}$ verificaram também que as medidas feitas com o mesmo dente, com um tempo mínimo de interlúdio, apresentaram menor divergência. Este achado é semelhante ao relatado por Shellhart et $\mathrm{al}^{5}$ que concluíram que a Análise de Bolton pode variar em $+2,2 \mathrm{~mm}$ quando se divide o arco em segmentos.

Embora a Análise de Bolton seja amplamente difundida e relativamente fácil de ser executada e aplicada, muitos ortodontistas não a utilizam para avaliação clínica, uma vez que o método é um pouco demorado quando os cálculos necessários são realizados manualmente. Na ortodontia contemporânea, os métodos computadorizados de diagnóstico estão se tornando uma alternativa vital para algumas medidas diagnósticas, tais como a relação de Bolton, tamanho do dente, largura do arco, overjet, sobremordida e comprimento do arco. As análises por computador requerem menos tempo ${ }^{21,22}$, permitindo além disso o mais fácil armazenamento e recuperação de dados, além de facilitar a comunicação entre profissionais. Porém. a precisão dos métodos tradicionais de avaliação devem ser resguardados.

\section{CONCLUSÃO}

Baseados na amostra estudada e na metodologia proposta neste estudo pode-se concluir que o método mais preciso foi o Paquímetro Digital, sendo este 3 vezes mais preciso que os demais métodos. Sua melhor correlação foi com o Compasso de Ponta Seca, que por sua vez obteve melhor precisão que o método Computadorizado.

\section{REFERÊNCIAS}

1. Cançado RH, Gonçalves Júnior W, Valarelli FP, Freitas KM, Crêspo JA. Association between Bolton discrepancy and Angle malocclusions. Braz Oral Res. 2015;29:1-6.

2. Akyalçin S, Dogan S, Dinçer B, Erdinc AM, Oncag G. Bolton tooth size discrepancies in skeletal Class I individuals presenting with different dental angle classifications. Angle Orthod. 2006;76(4):637-43.

3. Bolton WA. Disharmony in tooth size and its relation to the analysis and treatment of malocclusion. Angle Orthod. 1958;28(3):113-30.

4. Bolton WA. The clinical application of a tooth-size analysis. Am J Orthod. 1962;48(7):504-29.

5. Shellhart WC, Lange DW, Kluemper GT, Hicks EP, Kaplan AL. Reliability of the Bolton tooth-size analysis when applied to crowded dentitions. Angle Orthod. 1995;65(5):327-34.

6. Tomassetti JJ, Taloumis LJ, Denny JM, Fischer JR Jr. A comparison of 3 computerized Bolton tooth-size analyses with a commonly used method. Angle Orthod. 2001;71(5):351-7.

7. Ballard ML. Asymmetry in Tooth Size: a Factor in the Etiology, Diagnosis and Treatment of Malocclusion. Angle Orthod. 1944,14(3):67-70.
8. Doris JM, Bernard BW, Kuftinec MM, Stom D. A Biometric Study of Tooth Size and Dental Crowding. Am J Orthod. 1981;79(3):326-36.

9. Ramos AL, Suguino R, Terada HH, Furquim LZ, Silva Filho OG. Considerações Sobre Análise da Discrepância Dentária de Bolton e a Finalização Ortodôntica. Rev Dent Press Ortodon Ortop Facial. 1996; 1(2):86-106.

10. Moorrees CF, Reed RB. Correlation Among Crown Diameters of Human Teeth. Arch Oral Biol. 1964; 9:685-97.

11. Schirmer UR, Wiltshire WA. Manual and computeraided space analysis: a comparative study. Am J Orthod Dentofacial Orthop. 1997;112(6):676-80.

12. Halazonetis DJ. The Bolton ratio studied with the use of spreadsheets. Am J Orthod Dentofacial Orthop. 1996;109(2):215-9.

13. Begole EA, Cleall JF, Gorny HC. A computer program for the analysis of dental models. Comput Programs Biomed. 1979;10(3):261-70.

14. Bishara SE, Jakobsen JR, Abdallah EM, Fernandez Garcia A. Comparisons of mesiodistal and buccolingual crown dimensions of the permanent teeth in three populations from Egypt, Mexico, and the United States. Am J Orthod Dentofacial Orthop. 1989;96(5):416-22.

15. Yen CH. Computer-aided space analysis. J Clin Orthod. 1991;25(4):236-8.

16. Sakuda M, Tanne K, Yoshida K, Inoue H, Ohmae H, Tsuchiya $\mathrm{M}$, et al. Integrated information-processing system in clinical orthodontics: an approach with use of a computer network system. Am J Orthod Dentofacial Orthop. 1992;101(3):210-20.

17. Shahid F, Alam MK, Khamis MF. Intermaxillary tooth size discrepancy in a Pakistani population: A stereomicroscope versus digital caliper. Eur J Dent. 2016;10(2):176-82.

18. Yamamoto K, Toshimitsu A, Mikami T, Hayashi S, Harada R, Nakamura S. Optical measurement of dental cast profile and application to analysis of threedimensional tooth movement in orthodontics. Front Med Biol Eng. 1989;1(2):119-30.

19. Brandão MM, Sobral MC, Vogel CJ. Reliability of Bolton analysis evaluation in tridimensional virtual models. Dental Press J Orthod. 2015;20(5):72-7.

20. Sousa MVS, Vasconcelos EC, Janson G, Garib D, Pinzan A. Accuracy and reproducibility of 3dimensional digital model measurements. Am J Orthod Dentofacial Orthop. 2012;142(2):269-73.

21. Mullen SR, Martin CA, Ngan P, Gladwin M. Accuracy of space analysis with e-models and plaster models. Am J Orthod Dentofacial Orthop. 2007;132(3):346-52.

22. Proffit WR. Contemporary Orthodontics. 3rd ed. St. Louis: Mosby; 2000. p. 169-70. 
Arch Health Invest (2017) 6(2): 80-84

\section{CONFLITO DE INTERESSES}

Os autores declaram não haver conflitos de interesse.

\section{AUTOR PARA CORRESPONDÊNCIA}

\section{Renato Bigliazzi}

bigliazzi@foa.unesp.br

Submetido em 06/1 1/2016 Aceito em 05/12/2016 